\title{
Hypofractionated intensity-modulated radiotherapy with concurrent chemotherapy for elderly patients with locally advanced pancreatic carcinoma
}

Takahiro Iwai ${ }^{1}$, Michio Yoshimura ${ }^{1 *}$, Ryo Ashida ${ }^{1}$, Yoko Goto ${ }^{1}$, Takahiro Kishi ${ }^{1}$, Satoshi Itasaka ${ }^{1,2}$, Keiko Shibuya $^{1,3}$, Masashi Kanai ${ }^{4}$, Toshihiko Masui ${ }^{5}$, Akihisa Fukuda ${ }^{6}$, Hiroyoshi Isoda ${ }^{7}$, Masahiro Hiraoka ${ }^{1,8}$ and Takashi Mizowaki ${ }^{1}$

\begin{abstract}
Background: It is important to understand how elderly patients with locally advanced pancreatic carcinoma (LAPC) should be treated, since the number of elderly cancer patients will increase. However, the optimal treatment for elderly patients with LAPC remains unclear. The purpose of this study was to evaluate the efficacy and safety of hypofractionated intensity-modulated radiotherapy (IMRT) with concurrent gemcitabine for elderly patients with LAPC.

Methods: We retrospectively analysed the data from LAPC patients aged $\geq 75$ years treated with hypofractionated IMRT (48 Gy in 15 fractions) with concurrent weekly gemcitabine at our institution from February 2013 to December 2018. Overall survival (OS), progression-free survival (PFS), locoregional progression-free survival (LRPFS), distant metastasis-free survival (DMFS), and the pattern of recurrence and toxicity were analysed.

Results: Fifteen patients received treatment during the study period. The median age was 78 years (range 75-86 years), and the Eastern Cooperative Oncology Group (ECOG) performance status (PS) of all patients was 0-1. The median survival time (MST) and median PFS were 20.4 [95\% confidence interval (CI) 10.3-36.8] and 13.5 (95\% $\mathrm{Cl}$ 6.4-20.3) months, respectively, and the 1-year OS and PFS rates were $80.0 \%(95 \% \mathrm{Cl} 50-93.1 \%)$ and $66.7 \%(95 \% \mathrm{Cl}$ 37.5-84.6\%), respectively. The median LRPFS and median DMFS were $15.6(95 \% \mathrm{Cl} 6.4-36.8)$ and $14.9(95 \% \mathrm{Cl} 7.0-20.5)$ months, respectively, and the 1-year LRPFS and DMFS rates were $73.3 \%(95 \% \mathrm{Cl} 43.6-89.1 \%)$ and $66.7 \%(95 \% \mathrm{Cl} 37.5-$ $84.6 \%$ ), respectively. Non-haematologic grade 3 toxicity was observed in three cases, of which only one was induced by radiotherapy, whereas grade $4-5$ non-haematologic acute or late toxicities were not observed.
\end{abstract}

Conclusions: The OS and PFS of elderly patients with LAPC treated using hypofractionated IMRT with concurrent gemcitabine were favourable and without the occurrence of severe toxicity. This treatment strategy is feasible and promising for elderly LAPC patients with good PS.

${ }^{*}$ Correspondence: myossy@kuhp.kyoto-u.ac.jp

1 Department of Radiation Oncology and Image-Applied Therapy,

Graduate School of Medicine, Kyoto University, 54 Shogoin-Kawaharacho, Sakyo-ku, Kyoto 606-8507, Japan

Full list of author information is available at the end of the article

\section{Background}

Pancreatic carcinoma is a malignancy with a very poor prognosis. Approximately $30 \%$ of pancreatic carcinoma patients have unresectable locally advanced pancreatic carcinoma (LAPC) during diagnosis, with the prognosis of LAPC being poor [1]. The standard treatment for LAPC is chemotherapy (CTX) alone or concurrent chemoradiotherapy (CCRT). A previous study showed 
that CCRT was associated with decreasing local progression compared with CTX alone [2]. By contrast, the same trial described that the addition of radiotherapy (RT) to CTX did not improve overall survival (OS). However, an autopsy study showed that approximately $30 \%$ of patients with pancreatic cancer died because of locally destructive disease rather than distant metastasis [3]. Therefore, improving local control should be important for LAPC patients.

The treatment policy for LAPC remain controversial and the optimal treatment for elderly patients with LAPC is unclear. One of the reasons why the treatment for the elderly is still ill-defined is because the largest clinical trials have excluded elderly patients or have involved only a small number of them. For example, the trial that compared FOLFIRINOX with gemcitabine alone for metastatic pancreatic carcinoma registered patients aged 75 or younger [4], and the LAP07 trial that compared CCRT after induction CTX with CTX alone registered patients aged 71 or younger [2]. Therefore, it is meaningful to explore how elderly patients with LAPC should be treated, especially since the number of elderly patients with pancreatic cancer will increase within the next years [5].

Despite being promising for LAPC patients, the use of CCRT in pancreatic cancer could be problematic because of the anatomical relationship of the tumour with the surrounding organs. In particular, the gastrointestinal (GI) tracts such as the stomach or the duodenum are close to the pancreas, and excessive irradiation in these areas can cause radiation gastroenteritis, ulcer, perforation, or bleeding. Nevertheless, intensity-modulated radiotherapy (IMRT) can simultaneously reduce the dose to organ at risk (OAR), while assuring adequate target dose coverage compared to the conventional RT technique [6,7]. Our previous research showed that the treatment using hypofractionated IMRT with full dose gemcitabine had improved OS and locoregional progression-free survival (LRPFS) without increasing GI toxicities, compared to the treatment using conventional RT with low-dose gemcitabine [8]. In the context of a poor prognosis disease, it is also important to shorten hospitalization, as longterm hospitalization has been shown to result in cognitive decline or disuse syndrome especially in elderly patients $[9,10]$. For instance, hypofractionated RT is recommended for elderly patients with glioblastoma, a cancer that also has a very poor prognosis [11]. Hence, this treatment regimen of hypofractionated IMRT can be an ideal remedy for elderly patients with LAPC because of its low rate of GI toxicities and the short treatment period [12]. The purpose of this study was to investigate the efficacy and feasibility of hypofractionated IMRT with concurrent CTX for elderly patients with LAPC. To the best of our knowledge, this is the first study to evaluate the results of hypofractionated CCRT using IMRT for elderly LAPC patients.

\section{Methods}

This was a retrospective study that reviewed data from all LAPC patients aged 75 or above who have been treated with definitive CCRT from February 2013 to December 2018 at our institution. Written informed consent was obtained from all the patients. This study was approved by the institutional Review Board of Kyoto University Hospital (R1048). The consensus regarding unresectable LAPC and the indication for definitive CCRT was assessed by the cancer board of our institution, which involves specialists of surgery, gastroenterology, medical oncology, diagnostic radiology, and radiation oncology. The indication for definitive CCRT was decided after checking whether the organ function or ECOG PS of the patient were within the tolerable limits for this treatment. Patients with gastrointestinal mucosa tumour invasion were excluded. The definition of unresectability was celiac axis or superior mesenteric artery $>180$ degrees invasion, aortic invasion, or unreconstructible common hepatic artery or superior mesenteric vein, or portal occlusion [13]. Information regarding patient status, cancer stage, and treatment characteristics were obtained from the clinical records. The clinical stage was based on the 8th edition of the TNM classification for pancreas cancer of the Union for International Cancer Control (UICC) criteria [14]. Contrast-enhanced computed tomography (CT), dynamic magnetic resonance imaging (MRI) with Gadolinium-ethoxybenzyl-diethylenetriamine pentaacetic acid (Gd-EOB-DTPA), and ${ }^{18} \mathrm{~F}$-flurodeoxyglucose-positron emission tomography (FDG-PET) imaging were performed to determine the clinical stage. For FDG-PET, the early examination was performed at $1 \mathrm{~h}$ post-injection, followed by delayed examination at $90-360 \mathrm{~min}$ postinjection if necessary. All patients were hospitalised during CCRT and were monitored for acute haematopoietic, GI, and other toxicities.

After CCRT, the patients were periodically followed up and evaluated through physical and blood examinations; CT scans of the chest-abdomen-pelvis were also obtained. The patients were also monitored for late GI toxicities. If they had symptoms of gastroduodenal ulcer or haemorrhage, such as dizziness or stomach aches, then oesophagogastroscopy was performed. Depending on the timing of the side effects, toxicities were classified as during induction CTX, acute (from the initiation of the treatment to 28 days after CCRT), and late toxicities. The Common Terminology Criteria for Adverse Events version 4.0 was used for the assessment of all toxicities [15]. 


\section{Chemotherapy}

The treatment comprised induction CTX, CCRT, and maintenance CTX. The induction CTX regimen was based on gemcitabine $\left(1000 \mathrm{mg} / \mathrm{m}^{2}\right)$ that was administered intravenously once per week for 3 weeks, with 1 -week rest. On the other hand, the CCRT regimen is comprised of RT, with a total dose of 48 Gy delivered in 15 fractions using IMRT, and weekly gemcitabine $\left(1000 \mathrm{mg} / \mathrm{m}^{2}\right)$. For maintenance CTX, weekly gemcitabine $\left(1000 \mathrm{mg} / \mathrm{m}^{2}\right)$ was administered for 3-weeks, with 1-week rest. Maintenance CTX was repeated until tumour progression, worsening of the patient's condition, or the patient's refusal. The gemcitabine dose was reduced, or dose interval was extended if the standard dose could not be administered because of toxicity. Depending on the patient's medical condition, the regimen for induction and maintenance CTX were changed to gemcitabine in combination with nab-paclitaxel $\left(125 \mathrm{mg} / \mathrm{m}^{2}\right)$ for 3-weeks with 1-week rest at the discretion of the medical oncologist. When disease progression was detected, second-line CTX such as tegafur/gimeracil/oteracil potassium (S-1) or tegafur/uracil (UFT) was delivered if the patient's condition was good enough to receive CTX $[16,17]$.

\section{Radiotherapy}

For RT planning, 2-mm or $2.5-\mathrm{mm}$ slice contrastenhanced simulation $\mathrm{CT}$ was performed. To manage respiratory motion, breath-hold method, respiratory gating method, or dynamic tumour tracking method were adopted. The gross tumour volume (GTV) consisted of the primary tumour and metastatic lymph nodes. Contrast-enhanced CT was mainly used to determine the GTV, whereas MRI or FDG-PET were supplementally used. The clinical target volume (CTV) was defined as the GTV with a margin $5 \mathrm{~mm}$ in all directions plus the prophylactic area, which included the retropancreatic para-aortic lymph node and the neuroplexus involvement between the celiac axis and the superior mesenteric artery. The planning target volume (PTV) was CTV with a margin of $5 \mathrm{~mm}$ in all directions. The PTV-boost was the volume that subtracted the stomach plus 5 or $10 \mathrm{~mm}$, and the duodenum plus 3- or 5-mm margins from the PTV. This margin was adjusted using the techniques for managing respiratory motion. The prescription dose was specified as $\mathrm{D}_{95 \%}$ (the dose that covers $95 \%$ of the structure) to PTV-boost $=48$ Gy in 15 fractions and $\mathrm{D}_{98 \%}$ to PTV $\geq 36$ Gy using simultaneous integrated boost (SIB)IMRT technique. The dose constraints of OARs are listed in Table 1 [18]. If these dose constraints were not compatible with the dose prescriptions, dose prescriptions were decreased as $\mathrm{D}_{50 \%}$ to the PTV-boost $=48 \mathrm{~Gy}$. All
Table 1 Dose constraints for OAR

\begin{tabular}{ll}
\hline Structure & Constraints \\
\hline Stomach/duodenum & $\mathrm{V}_{45 \mathrm{~Gy}}<1 \mathrm{cc}$ \\
& $\mathrm{V}_{42 \mathrm{~Gy}}<5 \mathrm{cc}$ \\
& $\mathrm{V}_{39 \mathrm{~Gy}}<25 \mathrm{cc}$ \\
Stomach + PRV/duodenum +PRV & $\mathrm{V}_{39 \mathrm{~Gy}}<30 \mathrm{cc}$ \\
& $\mathrm{V}_{36 \mathrm{~Gy}}<45 \mathrm{cc}$ \\
Spinal cord & $\mathrm{D}_{\text {max }}<36 \mathrm{~Gy}$ \\
Spinal cord + PRV & $\mathrm{D}_{2 \mathrm{cc}}<39 \mathrm{~Gy}$ \\
Kidney (at least one) & $\mathrm{V}_{20 \mathrm{~Gy}}<30 \%$ \\
Liver & $\mathrm{D}_{\text {mean }}<30 \mathrm{~Gy}$ \\
\hline
\end{tabular}

OAR, organs at risk; $P R V$, planning organ at risk volume; $D_{\text {max }}$, the maximum dose to the structure volume; $D_{\text {mean }}$, the mean dose to the structure volume; $D_{2}$ ${ }_{{ }_{c c}}$ the maximum dose covering $\geq 2 \mathrm{cc}$ of the structure volume; $\mathrm{V}_{\mathrm{xx} G \mathrm{G}}$, the volume of the structure receiving $>x x$ Gy

patients were treated five times per week with $6 \mathrm{MV}$ or Flattening-Filter Free $10 \mathrm{MV}$ photons on a linear accelerator, Truebeam $^{\mathrm{TM}}$ (Varian Medical Systems, Inc, Palo Alto, California, USA) or Vero4DRT system (MHITM2000, Mitsubishi Heavy Industries, Ltd., Japan, and BrainLAB, Feldkirchen, Germany). Planning was performed with commercially available planning systems Eclipse $^{\mathrm{TM}}$ (Varian, Medical Systems, Palo Alto, California, USA) or iPlan ${ }^{\text {TM }}$ (Brainlab, Feldkirchen, Germany).

\section{Statistics}

OS was calculated from the starting date of induction CTX to the date of death by any cause and censored at the last follow-up visit for living patients. Progressionfree survival (PFS), LRPFS, and distant metastasis-free survival (DMFS) were calculated from the starting date of induction CTX to disease progression or death; to locoregional disease progression or death; and to the detection of first distant metastasis or death, respectively. Disease progression was defined as a proof of progressive disease on CT or FDG-PET imaging using the Response Evaluation Criteria in Solid Tumors. Statistical analyses were performed using EZR version 1.41 (Saitama Medical Center, Jichi Medical University, Saitama, Japan). The Kaplan-Meier techniques were used to estimate the OS, PFS, LRPFS, and DMFS.

\section{Results}

\section{Patient characteristics}

Between February 2013 and December 2018, 18 LAPC patients aged 75 or above were eligible for definitive CCRT as per our cancer board. All patients underwent induction CTX for 1-6 months prior to RT, and 15 out of 18 patients received CCRT. The remaining three patients could not receive CCRT because of disease progression, refusal of chemoradiotherapy, and refusal of CTX. Fifteen 
patients undergoing CCRT were included in the analysis, patient and tumour characteristics are summarised in Table 2. The radiation dose and fraction used among all patients were 48 Gy in 15 fractions. Regarding the specific prescription dose, 11 patients received the prescription dose and the dose to PTV-boost in 4 patients was decreased to $D_{50 \%} \geq 48$ Gy. The median age at diagnosis was 78 years (range 75-86 years), and nearly half of the patients were male. Eight (53.3\%) and 7 (46.7\%) patients showed PS of 0 and 1, respectively. According to the UICC Stage, all patients were in stage III. Primary tumours were located in the head/neck and the body/tail of the pancreas in 8 and 7 patients, respectively.

\section{Treatment outcomes and recurrence pattern}

At the time of analysis, the median follow-up period was 15.5 months, and three patients (20\%) were alive. The median survival time (MST) and median PFS were 20.4 [95\% confidence interval (CI) 10.3-36.8] and 13.5 (95\% CI 6.4-20.3) months, respectively. The 1-year OS and PFS rates were $80 \%$ (95\% CI 50-93.1\%) and 66.7\% (95\% CI 37.5-84.6\%), respectively (Fig. 1). The median LRPFS and median DMFS were 15.6 (95\% CI 6.4-36.8) and 14.9 (95\% CI 7.0-20.5) months, respectively, whereas the 1-year LRPFS and DMFS rates were $73.3 \%$ (95\% CI 43.6-89.1\%) and 66.7\% (95\% CI 37.5-84.6\%), respectively (Fig. 1). Among the 15 patients, 10 (66.7\%) exhibited some recurrences during the follow-up period. Two patients (13.3\%) had locoregional recurrence, six patients (40\%) had distant metastasis, and two patients (13.3\%) had both locoregional recurrence and distant metastasis when the first recurrence occurred. Peritoneal

Table 2 Patient and tumour characteristics $(n=15)$

\begin{tabular}{lccc}
\hline Characteristics & No & $\%$ & Years \\
\hline Age, median (range) & & & 78 (75-86) \\
Sex & 7 & 46.7 & \\
$\quad$ Male & 8 & 53.3 & \\
$\quad$ Female & & & \\
PS & 8 & 53.3 & \\
0 & 7 & 46.7 & \\
1 & & & \\
TNM stage (UICC 8th) & 14 & 93.3 & \\
CT4N0M0 stage III & 1 & 6.7 & \\
CT4N1M0 stage III & & & \\
Tumour location & 8 & 53.3 & \\
Head/uncus & 7 & 46.7 & \\
Body/tail & &
\end{tabular}

PS, performance status; UICC, Union for International Cancer Control; $\mathrm{T}$, tumour; $\mathrm{N}$, nodes; $\mathrm{M}$, metastasis dissemination was observed in most cases with distant metastasis. The recurrence patterns are summarised in Table 3.

\section{Variation of chemotherapy}

For induction CTX, 11 patients received gemcitabine alone, while four patients received gemcitabine in combination with nab-paclitaxel. The median period from the start of induction CTX to RT was 57 days (range 22-181 days). All patients received RT with concurrent gemcitabine. After RT, 14 patients underwent maintenance CTX except for one patient who had refused it. For maintenance CTX, 13 patients received gemcitabine and one patient received gemcitabine in combination with nab-paclitaxel until local recurrence or distant metastasis occurred. Because of worsened PS, adverse events, or patients' requests, dose was reduced, or dose interval of gemcitabine was extended for induction, concurrent, and adjuvant CTX, respectively. The variation and reduction of CTX are summarised in Table 4.

\section{Toxicities}

Data of acute and late adverse events for all 15 patients are shown in Table 5. For haematologic toxicity during induction CTX, Grade 3 or higher anaemia, neutropaenia and thrombocytopaenia occurred in 1 (6.7\%), 12 (80\%), 1 (6.7\%) patients, respectively. For acute toxicity, grade 3 or higher anaemia and neutropaenia were observed in 2 (13.3\%), and 5 (33.3\%) patients, respectively. Non-haematologic toxicity with grades 3 and 4 was observed during induction CTX and as acute toxicity. For late toxicity, Grade 3 diarrhoea and fatigue, arterial injury (pseudoaneurysm) were observed in $1(6.7 \%)$ patient. Diarrhoea and fatigue occurred when patients were administered S-1 or UFT for recurrence. Any other grade 3 or higher late toxicities were not observed.

\section{Discussion}

In elderly patients, aggressive treatment for malignant tumours is sometimes avoided because of age-associated functional decline, their severe comorbidities, or poor cognitive function [19]. This tendency is almost the same as that in pancreatic carcinoma [20-22]. In contrast, some studies reported that surgery or CTX could prolong OS for elderly patients with pancreatic carcinoma [21-23]. With regard to chemoradiotherapy for the elderly with LAPC, Miyamoto et al. suggested that the outcomes of patients treated with CCRT were similar to those of historical controls [24]. However, the patients in those studies were treated mainly with 5-FU and threedimensional conformal radiotherapy (3D-CRT). While Rakhra et al. showed the benefit of hypofractionated CRT for LAPC and Francesca et al. assessed the efficacy of 


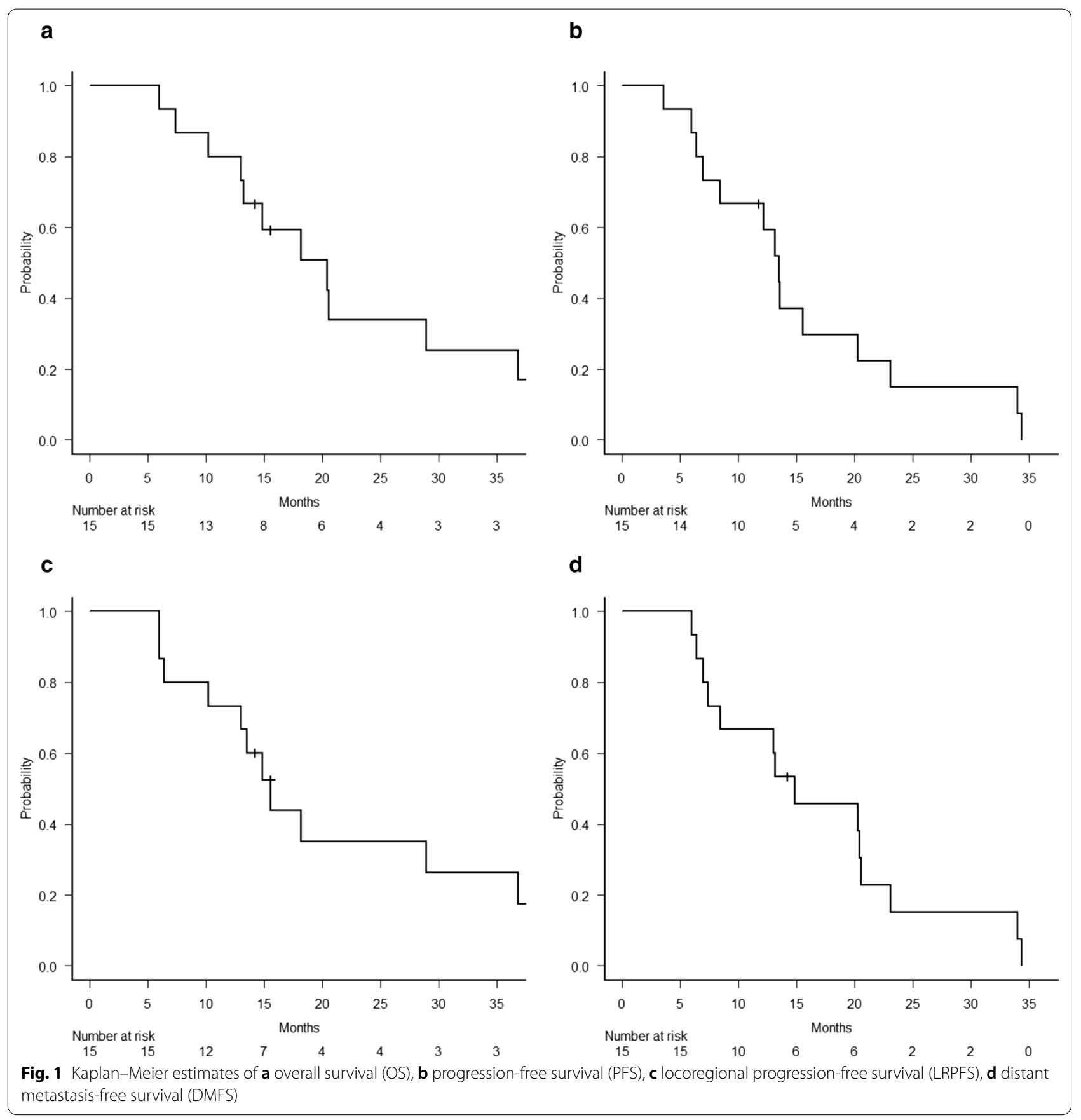

hypofractionated IMRT, no previous study focusing on elderly patients has been published $[25,26]$. Therefore, our study evaluated hypofractionated CCRT using IMRT with high-dose gemcitabine for the elderly.

Based on several reports, the MST ranged from 8.6 to 16.6 months and the median PFS ranged from 6.0 to 12.0 months in LAPC patients treated with CCRT among all ages [2, 27-31]. Among elderly patients, Miyamoto et al. [24] demonstrated that the MST was 8.6 months in 24 LAPC patients treated with CCRT using 3D-CRT. Several previous CCRT studies for LAPC are summarised in Table 6. Our study showed that the MST and median PFS were 20.4 and 13.5 months, respectively, which were not inferior to the results of previous reports on CCRT. Kuroda et al. reported that CTX alone, which consisted of mostly GEM-based regimens for elderly pancreatic carcinoma patients, resulted in 9.0 months (274 days) of MST among 519 pancreatic carcinoma 
Table 3 Pattern of recurrence $(n=15)$

\begin{tabular}{ll}
\hline Recurrence pattern & No. of patients (\%) \\
\hline Locoregional recurrence & $2(13.3)$ \\
Distant recurrence & $6(40)$ \\
Liver metastasis & $2(13.3)$ \\
Adrenal gland metastasis & $1(6.7)$ \\
Peritoneal dissemination & $2(13.3)$ \\
Multiple metastasis & $1(6.7)$ \\
Locoregional and distant recurrence & $2(13.3)$ \\
No evidence of recurrence & $5(33.3)$ \\
\hline
\end{tabular}

patients (approximately $28.3 \%$ of which were LAPC patients) [22]. However, it is difficult to compare our findings with that study because it included patients who had distant metastasis. Compared with these studies, our treatment resulted in favourable survival outcomes, in spite of the advanced age of our patients. Our previous study suggested that full dose gemcitabine and hypofractionated dose escalation with IMRT improved treatment outcomes, which could be applicable to elderly patients [8]. In addition, some recent studies demonstrated that high-dose radiation was a predictive factor for prolonged OS of patients with LAPC [31, 32]. In this study, we used a hypofractionated dose of 48 Gy in 15 fractions, of which the biological equivalent dose is almost equal to the conventional standard treatment dose (50.4-54 Gy in fractions of $1.8 \mathrm{~Gy}$ ), by calculating with an $\alpha / \beta$ value of 10 . However, considering the short overall treatment time, 48 Gy in 15 fractions is more potent than the conventional standard treatment dose and could contribute to better local control, which could lead to favourable survival outcomes.

As mentioned above, the OS and PFS were good; moreover, toxicity was also acceptable. Except one patient who had a pseudoaneurysm, grade 3 or higher non-haematologic adverse events induced by RT were not observed, and grade 3 non-hematologic toxicities because of CTX occurred in only two patients despite all patients being $\geq 75$ years (Table 5 ). The reason why our treatment strategy was less toxic is as follows: First, the dose to the surrounding normal organs was reduced with IMRT, which was demonstrated in our previous study [8]. Second, in induction CTX, the dose and the interval of concurrent or maintenance CTX could be adjusted. As seen in Table 3, many of the patients needed a reduction in their CTX dose or an extension of their CTX interval because of adverse events or their PS. In fact, haematologic side effects seemed less toxic during CCRT than induction CTX, especially in neutropaenia (Table 5). The major aim of induction CTX in our protocol was to select patients who have adequate tolerability of CCRT and secure the time for planning IMRT [33]. Furthermore, as described by some previous studies, using the adjusted dose of CTX in reference to the dose of induction CTX could contribute to an increase in the completion rate of

Table 4 Variation and reduction of chemotherapy (CTX)

\begin{tabular}{|c|c|c|}
\hline \multirow[t]{2}{*}{ Induction CTX } & \multicolumn{2}{|c|}{ No. of patients/total no. (\%) } \\
\hline & On schedule & Drug withdrawal \\
\hline Gemcitabine 1000 mg/m² & 5/15 (33.3) & 4/15 (26.7) \\
\hline Gemcitabine 800 mg/m² & $1 / 15(6.7)$ & $1 / 15(6.7)$ \\
\hline Gemcitabine and nab-paclitaxel & 0 & $4 / 15(26.7)$ \\
\hline \multirow[t]{2}{*}{ Concurrent CTX } & \multicolumn{2}{|c|}{ No. of patients/total no. (\%) } \\
\hline & On schedule & Drug withdrawal \\
\hline Gemcitabine 1000 mg/m² & $3 / 15(20)$ & $1 / 15(6.7)$ \\
\hline Gemcitabine 800 mg/m² & 2/15 (13.3) & $5 / 15(33.3)$ \\
\hline Gemcitabine 650 mg/m² & 0 & 2/15 (13.3) \\
\hline Gemcitabine 600 mg/m² & 0 & 2/15 (13.3) \\
\hline \multirow[t]{2}{*}{ Adjuvant CTX } & \multicolumn{2}{|c|}{ No. of patients/total no. (\%) } \\
\hline & On schedule & Drug withdrawal \\
\hline Gemcitabine 1000 mg/m² & $2 / 14(14.2)$ & $2 / 14(14.2)$ \\
\hline Gemcitabine 800 mg/m² & $1 / 14(7.1)$ & $5 / 14(35.7)$ \\
\hline Gemcitabine 650 mg/m² & 0 & $2 / 14(14.2)$ \\
\hline Gemcitabine 600 mg/m² & 0 & $1 / 14(7.1)$ \\
\hline Gemcitabine and nab-paclitaxel & 0 & $1 / 14(7.1)$ \\
\hline
\end{tabular}


Table 5 Toxicities $(n=15)$

\begin{tabular}{|c|c|c|c|c|}
\hline & Grade 1 & Grade 2 & Grade 3 & Grade 4 \\
\hline \multicolumn{5}{|c|}{ Toxicity during induction CTX } \\
\hline Anaemia & $2(13.3 \%)$ & $5(33.3 \%)$ & $1(6.7 \%)$ & 0 \\
\hline Neutropaenia & 0 & $2(13.3 \%)$ & $9(60 \%)$ & $3(20 \%)$ \\
\hline Thrombocytopaenia & $4(26.7 \%)$ & $5(33.3 \%)$ & $1(6.7 \%)$ & 0 \\
\hline Nausea & $3(20 \%)$ & 0 & 0 & 0 \\
\hline Fatigue & $8(53.3 \%)$ & $1(6.7 \%)$ & 0 & 0 \\
\hline Diarrhoea & $1(6.7 \%)$ & $1(6.7 \%)$ & 0 & 0 \\
\hline Rash & $3(20 \%)$ & $4(26.7 \%)$ & 0 & 0 \\
\hline \multicolumn{5}{|l|}{ Acute toxicity } \\
\hline Anaemia & $1(6.7 \%)$ & $6(40 \%)$ & $2(13.3 \%)$ & 0 \\
\hline Neutropaenia & 0 & $7(46.7 \%)$ & $3(20 \%)$ & $2(13.3 \%)$ \\
\hline Thrombocytopaenia & $5(33.3 \%)$ & $4(26.7 \%)$ & 0 & 0 \\
\hline Nausea & $4(26.7 \%)$ & $1(6.7 \%)$ & 0 & 0 \\
\hline Fatigue & $10(66.7 \%)$ & $1(6.7 \%)$ & 0 & 0 \\
\hline Diarrhoea & $1(6.7 \%)$ & $2(13.3 \%)$ & 0 & 0 \\
\hline Rash & $2(13.3 \%)$ & 0 & 0 & 0 \\
\hline \multicolumn{5}{|l|}{ Late toxicity } \\
\hline Fatigue & $4(26.7 \%)$ & $2(13.3 \%)$ & $1(6.7 \%)$ & 0 \\
\hline Diarrhoea & $1(6.7 \%)$ & $3(20 \%)$ & $1(6.7 \%)$ & 0 \\
\hline Arterial injury ${ }^{a}$ & 0 & 0 & $1(6.7 \%)$ & 0 \\
\hline Duodenal ulcer & $2(13.3 \%)$ & 0 & 0 & 0 \\
\hline
\end{tabular}

a Means pseudoaneurysm

CCRT $[28,34]$. As shown above, we adopted induction CTX before our CCRT using the IMRT protocol.

According to Table 3, the recurrence pattern was mainly distant metastasis, which was observed among 8 of 15 patients, whereas locoregional recurrence was observed among 4 of 15 patients. This trend was also reported in several previous studies [2, 35]. In the LAP07 study, OS was not prolonged compared to CTX alone, in spite of improving local control treated with CRT. The reason why local control could not improve OS may be because of the existence of occult metastasis for LAPC patients at the time of initial diagnosis; hence, systematic treatment is required for LAPC [1]. Conversely, local treatment could prolong OS among patients with no occult metastasis. Besides, local recurrence can cause obstructive cholangitis, duodenal obstruction, bleeding, or cancer pain. Therefore, improving local control by adding RT to CTX should be meaningful among LAPC patients, if this treatment could be tolerable. Since toxicity was not severe as we mentioned above, additional hypofractionated RT using IMRT may be good choice for elderly LAPC patients.

As this study was a retrospective and a single-arm analysis, there are several limitations. First, this analysis did not directly compare the results between CCRT and CTX alone or between IMRT and 3D-CRT for LAPC patients. Furthermore, we could not perform univariate or multivariate analyses for the prognostic factors because our sample size is small. A large number of randomised control trials are desirable to explore the most feasible therapy for elderly patients with LAPC. Second, our assessment of toxicities could be inaccurate. In particular, low-grade toxicity may have been underestimated because of the incomplete records on side effects, considering that this was a retrospective analysis. Nevertheless, we did not undercount severe toxicity, which should obviously be recorded, as it required additional medical care. Third, the PS of all patients in this study were 0 or 1 because these patients have been selected by our tumour board, and this result does not apply to all elderly patients. In other words, this treatment may be suitable for elderly patients with good PS who could tolerate chemoradiotherapy.

To the best of our knowledge, this is the first study to evaluate the outcomes and tolerability of hypofractionated CCRT using IMRT for elderly patients with LAPC. The results were favourable, and considering that the target was elderly people, hypofractionation may be meaningful because of its short treatment period. Since a long period of hospitalisation could induce disability such as cognitive decline or dementia in the elderly, short treatment is preferable. As the incidence of elderly patients with pancreatic carcinoma will increase, we should further investigate ideal treatments for LAPC patients, including other modalities.

\section{Conclusions}

In summary, hypofractionated IMRT with concurrent gemcitabine in elderly LAPC patients with good ECOG PS resulted in favourable local control and survival outcomes without severe frequent toxicity. This treatment strategy may be one of the appropriate treatment options for elderly patients with good PS. 


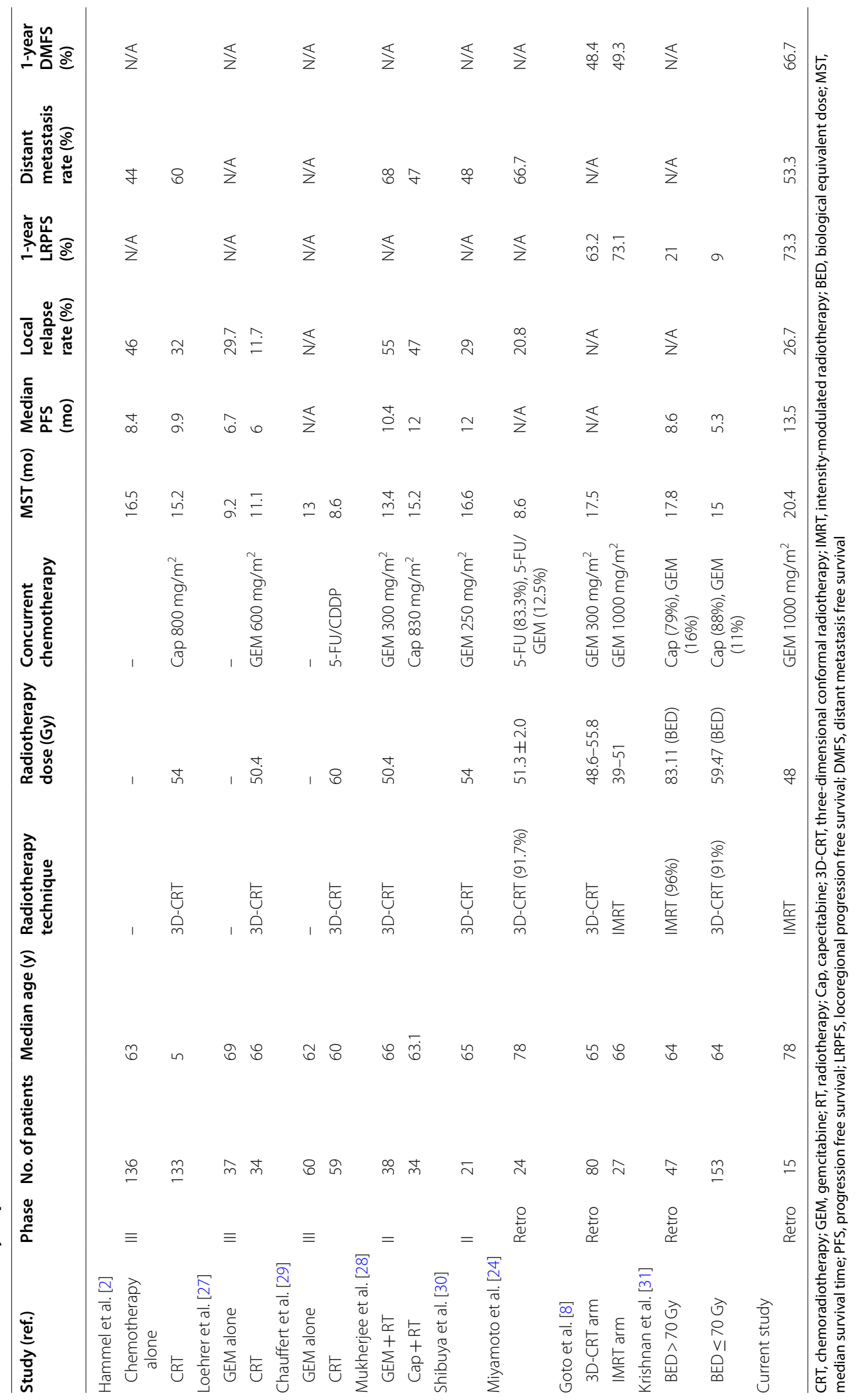




\begin{abstract}
Abbreviations
IMRT: Intensity-modulated radiotherapy; LAPC: Locally advanced pancreatic carcinoma; OS: Overall survival; PFS: Progression-free survival; LRPFS: Locoregional progression-free survival; DMFS: Distant metastasis-free survival; ECOG Eastern Cooperative Oncology Group; PS: Performance status; MST: Median survival time; Cl: Confidence interval; CTX: Chemotherapy; CCRT: Concurrent chemoradiotherapy; RT: Radiotherapy; GI: Gastrointestinal; OARs: Organ at risks; UICC: Union for International Cancer Control; CT: Computed tomography; MRI: Magnetic resonance imaging; Gd-EOB-DTPA: Gadolinium-ethoxybenzyl-diethylenetriamine pentaacetic acid; FDG-PET: ${ }^{18}$ F-flurodeoxyglucosepositron emission tomography; CTCAE: Common Terminology Criteria for Adverse Events; GTV: Gross Tumour volume; CTV: Clinical target volume; PTV: Planning target volume; SIB: Simultaneous integrated boost; 3D-CRT: Threedimensional conformal radiotherapy.
\end{abstract}

\section{Acknowledgements}

We would like to thank Akira Nakamura for patient treatment and data collection.

\section{Authors' contributions}

Tl performed designing the study, data analysis, and writing the final manuscript. MY, RA, YG, TK, MK, TM, AF, HI, MH, and TM contributed to patient treatment, analysis, and review the manuscript. SI and KS contributed to data collection, patient treatment, analysis, and review the manuscript. All authors read and approved the final manuscript.

\section{Funding}

This research was partially supported by the Japan Agency for Medical Research and development (AMED) under Grant No. JP20ck0106626.

\section{Availability of data and materials}

The datasets used and analysed for this study and provided upon reasonable request.

\section{Ethics approval and consent to participate}

The study was approved by the ethics committee of Kyoto University Hospital. Written consent to participate was obtained from the patient.

\section{Consent for publication}

Not applicable.

\section{Competing interests}

The authors declare that they have no competing interests.

\section{Author details}

${ }^{1}$ Department of Radiation Oncology and Image-Applied Therapy, Graduate School of Medicine, Kyoto University, 54 Shogoin-Kawaharacho, Sakyo-ku, Kyoto 606-8507, Japan. ${ }^{2}$ Department of Radiation Oncology, Kurashiki Central Hospital, Okayama, Japan. ${ }^{3}$ Department of Radiation Oncology, Graduate School of Medicine, Osaka City University, Osaka, Japan. ${ }^{4}$ Department of Clinical Oncology, Kyoto University Graduate School of Medicine, Kyoto, Japan. ${ }^{5}$ Department of Surgery, Kyoto University Graduate School of Medicine, Kyoto, Japan. ${ }^{6}$ Department of Gastroenterology and Hepatology, Kyoto University Graduate School of Medicine, Kyoto, Japan. ${ }^{7}$ Department of Diagnostic Imaging and Nuclear Medicine, Kyoto University Graduate School of Medicine, Kyoto, Japan. ${ }^{8}$ Department of Radiation Oncology, Japanese Red Cross Society Wakayama Medical Center, Wakayama, Japan.

Received: 18 July 2020 Accepted: 6 November 2020 Published online: 13 November 2020

\section{References}

1. Erdek MA, Fishman EK, Hruban RH. Recent progress in pancreatic cancer. CA Cancer J Clin. 2014;63(5):318-48. https://doi.org/10.3322/caac.21190.

2. Hammel P, Huguet F, Van Laethem JL, Goldstein D, Glimelius B, Artru P, Louvet $C$. Effect of chemoradiotherapy vs. chemotherapy on survival in patients with locally advanced pancreatic cancer controlled after 4 months of gemcitabine with or without erlotinib the LAP07 randomized clinical trial. JAMA J Am Med Assoc. 2016;315(17):1844-53. https://doi. org/10.1001/jama.2016.4324

3. Lacobuzio-Donahue CA, Fu B, Yachida S, Luo M, Abe H, Henderson CM, Laheru D. DPC4 gene status of the primary carcinoma correlates with patterns of failure in patients with pancreatic cancer. J Clin Oncol. 2009;27(11):1806-13. https://doi.org/10.1200/JCO.2008.17.7188.

4. Conroy T, Desseigne F, Ychou M, Bouché O, Guimbaud R, Bécouarn Y, Ducreux M. FOLFIRINOX versus gemcitabine for metastatic pancreatic cancer. N Engl J Med. 2011;364(19):1817-25. https://doi.org/10.1056/ NEJMoa1011923.

5. Higuera O, Ghanem I, Nasimi R, Prieto I, Koren L, Feliu J. Management of pancreatic cancer in the elderly. World J Gastroenterol. 2016;22(2):764-75. https://doi.org/10.3748/wjg.v22.i2.764.

6. Yovino S, Poppe M, Jabbour S, David V, Garofalo M, Pandya N, Regine WF. Intensity-modulated radiation therapy significantly improves acute gastrointestinal toxicity in pancreatic and ampullary cancers. Int J Radiat Oncol Biol Phys. 2011;79(1):158-62. https://doi.org/10.1016/j.jirob p.2009.10.043.

7. Colbert LE, Moningi S, Chadha A, Amer A, Lee Y, Wolff RA, Taniguchi CM. Dose escalation with an IMRT technique in 15 to 28 fractions is better tolerated than standard doses of 3DCRT for LAPC. Adv Radiat Oncol. 2017;2(3):403-15. https://doi.org/10.1016/j.adro.2017.02.004.

8. Goto Y, Nakamura A, Ashida R, Sakanaka K, Itasaka S, Shibuya K, Mizowaki T. Clinical evaluation of intensity-modulated radiotherapy for locally advanced pancreatic cancer. Radiat Oncol. 2018;13(1):1-9. https://doi. org/10.1186/s13014-018-1063-5.

9. Lutwak N. Cognitive decline after hospitalization in a community population of older persons. Neurology. 2012;79(5):486. https://doi.org/10.1212/ WNL.0b013e3182653b05.

10. Izquierdo M, Martínez-Velilla N, Casas-Herrero A, Zambom-Ferraresi F, Sáez De Asteasu ML, Lucia A, Rodríguez-Mañas L. Effect of exercise intervention on functional decline in very elderly patients during acute hospitalization: a randomized clinical trial. JAMA Intern Med. 2019;179(1):28-36. https://doi.org/10.1001/jamainternmed.2018.4869.

11. Phillips, C., Fay, M., Ch, B., Nishikawa, R., Cairncross, J. G., Roa, W., Investigators, T. Short-course radiation plus temozolomide in elderly patients with glioblastoma; 2017. p. 1027-1037. https://doi.org/10.1056/NEJMoa1611 977.

12. National Comprehensive Cancer Network. Central nervous system cancers V1. 2017, 123; 2016. Retrieved from https://www.nccn.org/profession als/physician_gls/pdf/cns.pdf.

13. Mizrahi JD, Surana R, Valle JW, Shroff RT. Pancreatic cancer. Lancet. 2020;395(10242):2008-20. https://doi.org/10.1016/S0140-6736(20)30974 $-0$.

14. Brierley JD, Gospodarowicz MK, Wittekind CE. The TNM classification of MALIGNANTTUMOURS. 8th ed. London: Wiley; 2017.

15. Common Terminology Criteria for Adverse Events v4.0. National Cancer Institute Cancer Therapy Evaluation Program (CTEP); 2009.

16. Morizane C, Okusaka T, Furuse J, Ishii H, Ueno H, Ikeda M, Suzuki E. A phase II study of S-1 in gemcitabine-refractory metastatic pancreatic cancer. Cancer Chemother Pharmacol. 2009;63(2):313-9. https://doi. org/10.1007/s00280-008-0741-7.

17. Feliu J, Mel R, Borrege P, López Gómez L, Escudero P, Dorta J, González Barón M. Phase II study of a fixed dose-rate infusion of gemcitabine associated with uracil/tegafur in advance carcinoma of the pancreas. Ann Oncol. 2002;13(11):1756-62. https://doi.org/10.1093/annonc/mdf286.

18. Goto Y, Ashida R, Nakamura A, Itasaka S, Shibuya K, Akimoto M, Hiraoka M. Clinical results of dynamic tumor tracking intensity-modulated radiotherapy with real-time monitoring for pancreatic cancers using a gimbal mounted linac. Oncotarget. 2018;9(34):23628-35. https://doi. org/10.18632/oncotarget.25310.

19. Talarico L, Chen G, Pazdur R. Enrollment of elderly patients in clinical trials for cancer drug registration: a 7-year experience by the US Food and Drug Administration. J Clin Oncol. 2004;22(22):4626-31. https://doi. org/10.1200/JCO.2004.02.175.

20. Krzyzanowska MK, Weeks JC, Earle CC. Treatment of locally advanced pancreatic cancer in the real world: Population-based practices and effectiveness. J Clin Oncol. 2003;21(18):3409-14. https://doi.org/10.1200/ JCO.2003.03.007. 
21. Sehgal R, Alsharedi M, Larck C, Edwards P, Gress T. Pancreatic cancer survival in elderly patients treated with chemotherapy. Pancreas. 2014;43(2):306-10. https://doi.org/10.1097/MPA.0000000000000091.

22. Kuroda T, Kumagi T, Yokota T, Azemoto N, Hasebe A, Seike H, Hiasa Y. Efficacy of chemotherapy in elderly patients with unresectable pancreatic cancer: a multicenter review of 895 patients. BMC Gastroenterol. 2017;17(1):1-7. https://doi.org/10.1186/s12876-017-0623-8.

23. Van Der Geest LGM, Besselink MGH, Van Gestel YRBM, Busch ORC, De Hingh IHJT, De Jong KP, Lemmens VEPP. Pancreatic cancer surgery in elderly patients: balancing between short-term harm and long-term benefit. A population-based study in the Netherlands. Acta Oncol. 2016;55(3):278-85. https://doi.org/10.3109/0284186X.2015.1105381.

24. Miyamoto DT, Mamon HJ, Ryan DP, Willett CG, Ancukiewicz M, Kobayashi WK, Hong TS. Outcomes and tolerability of chemoradiation therapy for pancreatic cancer patients aged 75 years or older. Int J Radiat Oncol Biol Phys. 2010;77(4):1171-7. https://doi.org/10.1016/j.jirobp.2009.06.020.

25. Rakhra S, Strauss JB, Robertson J, McGinn CJ, Kim T, Huang J, Small W. Hypofractionated conformal radiotherapy with concurrent full-dose gemcitabine versus standard fractionation radiotherapy with concurrent fluorouracil for unresectable pancreatic cancer: a multi-institution experience. J Gastrointes Cancer. 2016;47(2):196-201. https://doi.org/10.1007/ s12029-016-9821-9.

26. De Felice F, Benevento I, Bulzonetti N, Shima B, Rubini F, Marampon F, Tombolini V. Hypofractionated intensity-modulated radiotherapy in locally advanced unresectable pancreatic cancer: a pilot study. Curr Probl Cancer. 2019;43(5):495-503. https://doi.org/10.1016/j.currproblcancer 2019.04.003.

27. Loehrer PJ, Feng Y, Cardenes H, Wagner L, Brell JM, Cella D, Benson AB. Gemcitabine alone versus gemcitabine plus radiotherapy in patients with locally advanced pancreatic cancer: An Eastern Cooperative Oncology Group trial. J Clin Oncol. 2011;29(31):4105-12. https://doi.org/10.1200/ JCO.2011.34.8904

28. Mukherjee S, Hurt CN, Bridgewater J, Falk S, Cummins S, Wasan H, Maughan T. Gemcitabine-based or capecitabine-based chemoradiotherapy for locally advanced pancreatic cancer (SCALOP): a multicentre, randomised, phase 2 trial. Lancet Oncol. 2013;14(4):317-26. https://doi. org/10.1016/S1470-2045(13)70021-4.

29. Chauffert, B., Mornex, F., Bonnetain, F., Rougier, P., Mariette, C., Bouche, O., Maingon, P. Phase III trial comparing intensive induction gemcitabine with gemcitabine alone for locally advanced unresectable pancreatic cancer. Definitive results of the 2000-01 FFCD/SFRO study (May); 2008. p. 1592-1599. https://doi.org/10.1093/annonc/mdn281.

30. Shibuya K, Oya N, Fujii T, Doi R, Nakamura A, Matsuo Y, Hiraoka M. Phase II study of radiation therapy combined with weekly low-dose gemcitabine for locally advanced, unresectable pancreatic cancer. Am J Clin Oncol Cancer Clin Trials. 2011;34(2):115-9. https://doi.org/10.1097/COC.0b013 e3181c4c7a8

31. Krishnan S, Chadha AS, Suh Y, Chen HC, Rao A, Das P, Crane CH. Focal radiation therapy dose escalation improves overall survival in locally advanced pancreatic cancer patients receiving induction chemotherapy and consolidative chemoradiation. Int J Radiat Oncol Biol Phys. 2016;94(4):755-65. https://doi.org/10.1016/j.jirobp.2015.12.003.

32. Choi SH, Park SW, Seong J. A nomogram for predicting survival of patients with locally advanced pancreatic cancer treated with chemoradiotherapy. Radiother Oncol. 2018;129(2):340-6. https://doi.org/10.1016/j. radonc.2018.08.006.

33. Huguet F, André T, Hammel P, Artru P, Balosso J, Selle F, Louvet C. Impact of chemoradiotherapy after disease control with chemotherapy in locally advanced pancreatic adenocarcinoma in GERCOR phase II and III studies. J Clin Oncol. 2007;25(3):326-31. https://doi.org/10.1200/ JCO.2006.07.5663.

34. Krishnan S, Rana V, Janjan NA, Varadhachary GR, Abbruzzese JL, Das $\mathrm{P}, \mathrm{Crane} \mathrm{CH}$. Induction chemotherapy selects patients with locally advanced, unresectable pancreatic cancer for optimal benefit from consolidative chemoradiation therapy. Cancer. 2007;110(1):47-55. https://doi. org/10.1002/cncr.22735.

35. Hurt CN, Falk S, Crosby T, McDonald A, Ray R, Joseph G, Mukherjee S. Long-term results and recurrence patterns from SCALOP: A phase II randomised trial of gemcitabine- or capecitabine-based chemoradiation for locally advanced pancreatic cancer. Br J Cancer. 2017;1 16(10):1264-70. https://doi.org/10.1038/bjc.2017.95.

\section{Publisher's Note}

Springer Nature remains neutral with regard to jurisdictional claims in published maps and institutional affiliations.
Ready to submit your research? Choose BMC and benefit from:

- fast, convenient online submission

- thorough peer review by experienced researchers in your field

- rapid publication on acceptance

- support for research data, including large and complex data types

- gold Open Access which fosters wider collaboration and increased citations

- maximum visibility for your research: over $100 \mathrm{M}$ website views per year

At BMC, research is always in progress.

Learn more biomedcentral.com/submissions 